\title{
Melatonin prevents doxorubicin-induced cardiotoxicity through suppression of AMPKa2-dependent mitochondrial damage
}

\author{
Goowon Yang ${ }^{1}$, Minhyeok Song ${ }^{1}$, Dang Hieu Hoang ${ }^{1}$, Quynh Hoa Tran', Wonchae Choe ${ }^{1}$ Insug Kang ,
} Sung Soo Kim ${ }^{1}$ and Joohun Ha (1)

\begin{abstract}
The clinical application of doxorubicin, one of the most effective anticancer drugs, has been limited due to its adverse effects, including cardiotoxicity. One of the hallmarks of doxorubicin-induced cytotoxicity is mitochondrial dysfunction. Despite intensive research over recent decades, there are no effective approaches for alleviating doxorubicin-induced cytotoxicity. Melatonin, a natural hormone that is primarily secreted by the pineal gland, is emerging as a promising adjuvant that protects against doxorubicin-induced cytotoxicity owing to its pharmaceutical effect of preserving mitochondrial integrity. However, the underlying mechanisms are far from completely understood. Here, we provide novel evidence that treatment of H9c2 cardiomyoblasts with doxorubicin strongly induced AMP-activated protein kinase a2 (AMPKa2), which translocated to mitochondria and interfered with their function and integrity, ultimately leading to cellular apoptosis. These phenomena were significantly blocked by melatonin treatment. The levels of AMPKa2 in murine hearts were tightly associated with cardiotoxicity in the context of doxorubicin and melatonin treatment. Therefore, our study suggests that the maintenance of mitochondrial integrity is a key factor in reducing doxorubicin-induced cytotoxicity and indicates that AMPKa2 may serve as a novel target in the design of cytoprotective combination therapies that include doxorubicin.
\end{abstract}

\section{Introduction}

Doxorubicin is one of the most potent chemotherapeutic agents and is widely used for the treatment of various cancers and hematological malignancies. However, the more widespread use of this agent is limited owing to its severe adverse effects, including cardiotoxicity, neurological disturbances, and bone marrow aplasia ${ }^{1,2}$. Decades of studies on doxorubicin have implicated multifactorial processes in the development of doxorubicin-induced cytotoxicity, but recent studies suggest that mitochondrial dysfunction is a distinctive hallmark of doxorubicininduced cytotoxicity ${ }^{3,4}$.

To alleviate doxorubicin-induced toxicity, researchers have tested a number of strategies, including the

\footnotetext{
Correspondence: Joohun Ha (hajh@khu.ac.kr)

'Department of Biochemistry and Molecular Medicine, Graduate School,

Biomedical Science Institute, Kyung Hee University, Seoul, Korea
}

administration of antioxidants and/or antiapoptotic agents, in both in vitro and in vivo models of doxorubicininduced cytotoxicity, but most of these trials have failed to translate into clinical benefits ${ }^{5-7}$. As a result, there are no effective approaches for alleviating doxorubicin-induced cytotoxicity despite intensive research over recent decades. Melatonin is a natural hormone that is primarily secreted by the pineal gland and functions as a major regulator of circadian rhythms in humans ${ }^{8}$. Melatonin also plays a variety of biological roles as a modulator of mood, sexual behavior and sleep; low levels or a deficiency of melatonin are also associated with Parkinson's disease, Alzheimer's disease, epilepsy, ischemic injury, diabetes, and even cancer ${ }^{8,9}$. Melatonin has emerged as a promising adjuvant that protects against doxorubicin-induced cytotoxicity, as highlighted by various studies and clinical trials that have demonstrated its cardioprotective effects 
against several chemotherapeutic agents ${ }^{10-12}$. These beneficial effects of melatonin are largely associated with its ability to prevent mitochondrial dysfunction and its strong antioxidant properties ${ }^{13-15}$. Moreover, melatonin exhibits low toxicity and easily enters cells owing to its good solubility in both aqueous and organic phases and its highly lipophilic properties ${ }^{13}$. Thus, melatonin shows great promise for use in this therapeutic context.

In the present study, we examined the mechanisms by which melatonin attenuates doxorubicin-induced cytotoxicity, revealing a novel role of AMPK $\alpha 2$ in the mitochondria of $\mathrm{H} 9 \mathrm{c} 2$ cardiomyoblasts and mouse hearts. AMPK is composed of a catalytic subunit $(\alpha)$ and two regulatory subunits ( $\beta$ and $\gamma$ ), each with multiple isoforms, and AMPK plays a central role in the regulation of energy homeostasis ${ }^{16-20}$. Regarding the catalytic subunit, $\alpha 1$ is ubiquitously expressed in all tissues, but $\alpha 2$ is highly expressed in metabolically active tissues, such as the heart, skeletal muscle, and liver; $\alpha 2$ has also been implicated in the regulation of carbohydrate and lipid metabolism ${ }^{21-23}$. In a previous study, we described a novel role for AMPK $\alpha 2$ in the context of the treatment of mouse embryonic fibroblasts (MEFs) with doxorubicin, showing that AMPK 22 expression is robustly induced by doxorubicin at the transcriptional level via the transcription factor E2F1 and subsequently contributes to apoptosis ${ }^{24}$. However, the features of AMPK $\alpha 2$ that are responsible for these proapoptotic properties remain unknown. Here, we provide novel evidence that AMPK $\alpha 2$ exerts a proapoptotic effect in the context of doxorubicin treatment by interfering with mitochondrial integrity in H9c2 cardiomyoblasts and mouse hearts. Notably, we found that these effects were effectively blocked by melatonin.

\section{Materials and methods \\ Reagents and antibodies}

Dulbecco's modified Eagle's medium (DMEM), F-12 (1:1), and bovine serum (BS) were purchased from Gibco (Grand Island, NY). Fetal bovine serum (FBS) was purchased from Corning (Corning, NY). Melatonin, doxorubicin, H2DCF-DA, intracellular ATP assay kits, N-acetyl cysteine, and 4-P-PDOT (4-phenyl-2-propionamidotetralin) were purchased from Sigma-Aldrich (St. Louis, MO). MTT thiazolyl blue and D-luciferin were purchased from Duchefa Biochemie (Haarlem, the Netherlands). 4',6-Diamidino-2-phenylindole (DAPI), enhanced chemiluminescence reagent, antibodies against GAPDH, E2F1, c-Myc, and voltage-dependent anion channel (VDAC), MitoTracker red, and control IgG antibodies were purchased from Santa Cruz Biotechnology (Santa Cruz, CA). AntiAMPK $\alpha 2$ and anti-AMPK $\alpha 1$ antibodies were purchased from R\&D Systems (Minneapolis, MN). MitoTracker green and anti-phospho-H2A.X (S139), anti-phospho-AMPKa (Thr172), anti-phospho-acetyl-CoA carboxylase (ACC)
(S79), anti-ACC, anti-phospho-mitochondria fission factor (MFF) (S146), and anti-MFF antibodies were purchased from Cell Signaling Technology (Danvers, MA). MitoSOX Red was purchased from Invitrogen (Carlsbad, CA). The FITC-annexin V apoptosis kit was purchased from DB BioScience (San Diego, CA). The Total OXPHOS Rodent WB Antibody Cocktail was purchased from Abcam (Cambridge, MA).

\section{Cell culture}

H9c2 (rat cardiomyoblast) cells were purchased from American Type Culture Collection (ATCC CRL-1446) in 2012 and maintained in DMEM and F-12 (1:1) supplemented with $10 \%$ BS. H9c2 cells fuse to form multinucleated myotubes in response to low serum, and a recent characterization was performed in 2017. Microscopic analysis revealed that these cells formed multinucleated myotubes when cultured in media containing $1 \%$ horse serum, and RT-PCR analysis showed an increase in the expression of acetyl-CoA carboxylase $\beta$, which is a specific marker of myotube formation ${ }^{25}$. AMPK wild-type (WT) and AMPK knockout MEFs were generated by Benoit Viollet (INSERN, France) in $2006^{26}$ and generously provided to us in 2008. Ampka WT, $A m p k \alpha 2^{-1-}$, Ampk $\alpha 1^{-/-}$, and Ampk double knockout $\left(\alpha 1^{-/-} \alpha 2^{-/-}\right)$ MEFs were maintained in DMEM supplemented with $10 \%$ FBS. We characterized these cells according to the presence of AMPK isoforms and simian virus 40 large $T$ antigen using Western blot and RT-PCR in 2018. All the cells were supplemented with $1 \%$ penicillin/streptomycin and cultured at $37{ }^{\circ} \mathrm{C}$ in a humidified environment with $95 \%$ air and $5 \% \mathrm{CO}_{2}$.

\section{Stable cell transfection}

When the density of the H9c2 myocytes reached $50-60 \%$, the cells were transfected with the expression vector pcDNA 3.1 encoding c-Myc-tagged wild-type (WT) or dominant negative (DN, K45A) forms of AMPK $\alpha 2$ using Lipofectamine 3000 (Invitrogen, Carlsbad, CA, USA). The cells were selected for 7 days in the presence of G418 $(1 \mathrm{mg} / \mathrm{mL})$, and the selected cells were used for the subsequent experiments.

\section{DNA plasmids}

The promoter region of the human AMPK $\alpha 2$ gene $(-2.7 \mathrm{~kb})$ was amplified by PCR from HEK293 genomic DNA and cloned into the pGL3-basic reporter vector. The [E2F]x4-Luc reporter construct was generously provided by Dr. Young-Chae Chang (The Catholic University, School of Medicine, Daegu, Korea). AMPK $\alpha 2$ wild-type (WT) and AMPK 2 2 dominant-negative (DN, D157A) forms were generated by PCR and cloned into the pCMV vector. The dsRed-mito plasmid was generously provided by Dr. Youngmi Kim Pak (Kyung hee University, School 
of Medicine, Seoul, Korea). Mito-ABKAR was a gift from Takanari Inoue and Jin Zhang (Addgene plasmid \#61509). Tom20-mChF-AIP and mChF-AIP were gifts from Takanari Inoue (Addgene plasmids \#61512 and \#61527).

\section{Cell viability assay}

Cell viability was measured in 96-well plates using a quantitative colorimetric assay with 3-(4,5-dimethylthiazol-2-yl)-2,5-diphenyltetrazolium bromide (MTT), which is an indicator of mitochondrial activity in live cells. Briefly, $30 \mu \mathrm{L}$ MTT (final concentration, $0.5 \mathrm{mg}$ / $\mathrm{mL}$ ) was added to the medium at the indicated times after treatment and then incubated at $37^{\circ} \mathrm{C}$ for $4 \mathrm{~h}$. The MTT solution was removed, and $100 \mu \mathrm{L}$ DMSO was added to each well. The absorbance of each well was measured at $540 \mathrm{~nm}$ using a microplate reader (BioTek, Winooski, VT).

\section{Western blot analysis and immunoprecipitation}

Cells were lysed in lysis buffer $(50 \mathrm{mM}$ Tris- $\mathrm{HCl}, \mathrm{pH}$ 7.4, $150 \mathrm{mM} \mathrm{NaCl}, 1 \% \mathrm{NP} 40,2 \mathrm{mM}$ EDTA, $10 \mathrm{mM} \mathrm{NaF}$, $2 \mathrm{mM} \mathrm{Na} 3 \mathrm{VO} 4)$. The protein concentrations in the total lysates were determined by the Bradford method (Bio-Rad, Hercules, CA, USA). Twenty micrograms of protein from the total lysates was subjected to SDS-PAGE and transferred to Immobilon-P membranes (Millipore, Bedford, MA). Then, the membranes were incubated with primary antibodies in blocking solution (5\% skim milk). The proteins were visualized using the ECL detection system. For the immunoprecipitation experiments, cell lysates were precleared with protein G/A beads and subsequently incubated for $1-2 \mathrm{~h}$ with protein G/A beads covalently coupled with anti-AMPK $\alpha 2$ or anti-c-Myc. The immune complexes were washed three times with cell lysis buffer. The eluted samples and whole lysates were resolved by SDS-PAGE, and the proteins were detected by western blot using the indicated antibodies. Quantification of western blots was performed by using ImageJ software.

\section{Luciferase assay}

H9c2 cells were cotransfected with $0.5 \mu \mathrm{g}$ of the reporter vector. The empty pcDNA vector was used to adjust the total amount of DNA, and $0.5 \mu \mathrm{g}$ of pCMVEGFP expression plasmid was used as the internal control. Luciferase activity was determined using a luciferase assay kit (Promega) according to the manufacturer's instructions. The relative luciferase activity was normalized against the GFP fluorescence intensity.

\section{Fluorescence-activated cell sorting (FACS) analysis}

Fluorescence was determined by flow cytometry (Beckman, Pasadena, CA). MitoTracker green, H2DCFDA, and Annexin V-FITC fluorescence was measured by excitation at $488 \mathrm{~nm}$ and emission collection at $525 \mathrm{~nm}$. Propidium iodide (PI), MitoTracker red, and MitoSOX Red fluorescence was measured by using excitation and emission wavelengths of 540 and $580 \mathrm{~nm}$, respectively.

\section{Confocal microscopy}

H9c2 cells were plated on glass coverslips and treated the following day with doxorubicin, with or without melatonin. The cells were fixed by incubating with $4 \%$ paraformaldehyde for $20 \mathrm{~min}$ and washed with phosphatebuffered saline (PBS). After permeabilization with $0.1 \%$ Triton X-100 in PBS for 3 min at room temperature, the cells were incubated with a blocking solution for $30 \mathrm{~min}$ at $37^{\circ} \mathrm{C}$. Solutions of anti-AMPK $\alpha 2$ (1:500) primary antibodies were added to the cells and incubated overnight at $4{ }^{\circ} \mathrm{C}$. Thereafter, the cells were washed with PBS and then incubated with 1:500 dilutions of FITC- or Texas redconjugated secondary antibodies for $2 \mathrm{~h}$ at $37^{\circ} \mathrm{C}$. MitoTracker was added before fixation, and DAPI was added after incubation with secondary antibodies. For the Förster resonance energy transfer (FRET) experiments, H9c2 cells were transfected with the mito-ABKAR plasmid and then treated the following day with doxorubicin and/or melatonin. Cyan fluorescent protein (CFP) was excited at $433 \mathrm{~nm}$, and its emitted fluorescence was collected at $480 \mathrm{~nm}$. FRET images were obtained by exciting yellow fluorescent protein (YFP) at $508 \mathrm{~nm}$ and collecting its emitted fluorescence at $527 \mathrm{~nm}$. The lengths of mitochondria were calculated from each YFP image using ImageJ software (image.nih.gov/ij/), as previously described $^{27}$, and the FRET fluorescence intensity was analyzed. Images of the cells were acquired using an LSM 510 confocal microscope (Carl Zeiss, Jena, Germany).

\section{RT-PCR}

Total RNA was extracted from cells using TRIzol reagent (Invitrogen). cDNA was synthesized from $2 \mathrm{mg}$ of total RNA using M-MLV reverse transcriptase (Fermentas, Hanover, MD, USA). The specific primers used for RT-PCR were as follows: AMPK $\alpha 2,5^{\prime}$-AGC TCG CAG TGG CTT ATC AT-3' (sense) and 5'-GGG GCT GTC TGC TAT GAG AG-3' (anti-sense); and GAPDH, 5'-A GA CAG CCG CAT CTT CTT GT-3' (sense) and $5^{\prime}$-CTT GCC GTG GGT AGA GTC AT-3' (anti-sense). The PCR products were analyzed by agarose gel electrophoresis and visualized using ethidium bromide. The signals were quantified using Image) software (National Institutes of Health (NIH), Bethesda, MD, USA).

\section{Measurement of mitochondrial DNA content}

Total genomic DNA was extracted from H9c2 cells and used as a template for the amplification of mitochondrially encoded cytochrome $\mathrm{C}$ oxidase II (MTCO2) and 
$18 \mathrm{~S}$ rRNA by real-time quantitative polymerase chain reaction (qPCR). The following specific primer pairs were used: MTCO2, 5'-GCT TAC AAG ACG CCA CAT CA-3' (sense) and 5'-GAA TTC GTA GGG AGG GAA GG-3' (anti-sense); 18S rRNA, 5'-CGC GGT TCT ATT TTG TTG GT-3' (sense) and $5^{\prime}$-AGT CGG CAT CGT TTA TGG TC-3' (anti-sense). qPCR was performed using a 7500 Real-Time PCR System (Applied Biosystems, Branchburg, NJ, USA) with SYBRGreen PCR Master Mix (Applied Biosystems). A total of 40 thermal cycles were used for the PCR, and the expression of MTCO2 was analyzed using an absolute quantification method and normalized to the levels of $18 \mathrm{~S}$ rRNA.

\section{Measurement of intracellular ATP levels}

Cells were seeded in 24-well plates and stimulated as indicated. The intracellular ATP content was measured using an ATP Determination Kit (Sigma-Aldrich) in accordance with the manufacturer's instructions.

\section{Animal experiments}

All the mice were purchased from Japan SLC (Shizuoka, Japan) and housed at $21 \pm 2{ }^{\circ} \mathrm{C}$ and $50 \% \pm 5 \%$ relative humidity with a 12 -h light/12-h dark cycle. The mice were randomly divided into 4 groups $(n=5 /$ group): sham, doxorubicin, melatonin, and doxorubicin + melatonin. Melatonin $(1 \mathrm{mg} / \mathrm{kg})$ and/or doxorubicin $(2.5 \mathrm{mg} / \mathrm{kg})$ were intraperitoneally injected into 4-weekold mice (Slic: ICR, male) every 2 days for 2 weeks. After 2 weeks of treatment, heart tissues were isolated, and five-micron-thick sections were subjected to H\&E staining, Masson trichrome staining, immunohistochemical analysis (IHC) of AMPK $\alpha 2$, and colorimetric TUNEL assay (Promega Corp., Madison, WI). The thickness of the myofibrillum was measured as previously described ${ }^{28}$. The TUNEL-positive nuclei (dark brown) in the heart tissues were detected using a normal white light microscope (Olympus, Tokyo, Japan). Quantification was performed by counting the number of TUNEL-positive cells in at least five random fields. The animal protocol was approved by the Institutional Animal Care and Use Committee of Kyung Hee University (KHSASP-19-297).

\section{Data and statistical analysis}

The results are expressed as the means \pm SEMs. The statistical significance of the data were analyzed by oneway analysis of variance (ANOVA) using the $\mathrm{R}$ program suite (version 3.2.4; http://www.r-project.org). By convention, a $p$-value $<0.05$ was considered statistically significant. Individual $p$-values are indicated in the figures (" $p$ $<0.05$; $* *<0.01)$. Each experiment was repeated at least twice with three samples each to ensure statistical significance.

\section{Results}

Melatonin blocks doxorubicin-induced AMPKa2 expression, which has proapoptotic properties in $\mathrm{H} 9 \mathrm{c} 2$ cardiomyoblasts

We first examined H9c2 cardiomyoblast cell viability following treatment with doxorubicin and melatonin. The results showed that melatonin alone did not cause toxicity, but it significantly inhibited doxorubicin-induced cytotoxicity (Fig. 1a). In a previous study, we observed that doxorubicin simultaneously induced the expression of AMPK $\alpha 2$, E2F1, and apoptosis markers, including cleaved PARP and caspase-3, within $24 \mathrm{~h}$ in $\mathrm{H} 9 \mathrm{c} 2$ cells $^{24}$. Melatonin also blocked the induction of AMPK $\alpha 2$ by doxorubicin (Fig. 1b), which occurred at the transcriptional level, as evidenced by increases in AMPK $\alpha 2$ mRNA levels and promoter activity (Fig. 1c, d). Consistent with this finding, doxorubicin also induced the expression of E2F1, an upstream transcription factor for AMPK $\alpha 2$ (Fig. 1c, e). Overexpression of WT AMPK $\alpha 2$ (AMPK $\alpha 2 W T)$ potentiated doxorubicin-induced apoptosis, whereas a dominant-negative form of AMPK $\alpha 2$ (AMPK $\alpha 2 D N)$ suppressed this phenomenon (Fig. 1f), indicating that AMPK $\alpha 2$ possesses proapoptotic properties under these conditions, in accordance with a previous report ${ }^{24}$. Collectively, these results indicate that doxorubicin induces AMPK $\alpha 2$, which has proapoptotic properties, and that melatonin suppresses this expression. Throughout the subsequent studies, cells were treated with doxorubicin $(1 \mu \mathrm{M})$ and melatonin $(1 \mathrm{mM})$ for $24 \mathrm{~h}$.

\section{AMPKa2 translocates to mitochondria and disrupts their function in response to doxorubicin in $\mathrm{H} 9 \mathrm{c} 2$ cells and MEFs, and melatonin blocks these phenomena}

We next examined whether AMPK $\alpha 2$ plays a specific role in the regulation of mitochondrial function and integrity. Doxorubicin caused severe mitochondrial damage in H9c2 cells, as indicated by mitochondrial membrane potential (Fig. 2a), cellular and mitochondrial ROS levels (Fig. 2b), mitochondrial OXPHOS complex protein levels (Fig. 2c), mitochondrial DNA content (Fig. 2d), and cellular ATP levels (Fig. 2e). Each of these signs of mitochondrial damage was effectively blocked by melatonin. Under these conditions, the overexpression of AMPK $\alpha 2$ WT potentiated the mitochondrial damage induced by doxorubicin, whereas AMPK $\alpha 2 \mathrm{DN}$ reduced this mitochondrial damage. Western blot analysis of mitochondrial fractions (Fig. 2f) and confocal microscopy analysis (Fig. 2g) revealed that a significant portion of AMPK $\alpha 2$ was translocated to mitochondria following doxorubicin treatment and that this translocation was blocked by melatonin.

We further examined the specific role of AMPK $\alpha 2$ in the mitochondria of WT, Ampk $\alpha 1^{-/-}, A m p k \alpha 2^{-1-}$, and Ampk $\alpha 1^{-/-} \alpha 2^{-/-}$double-knockout MEFs. The total 


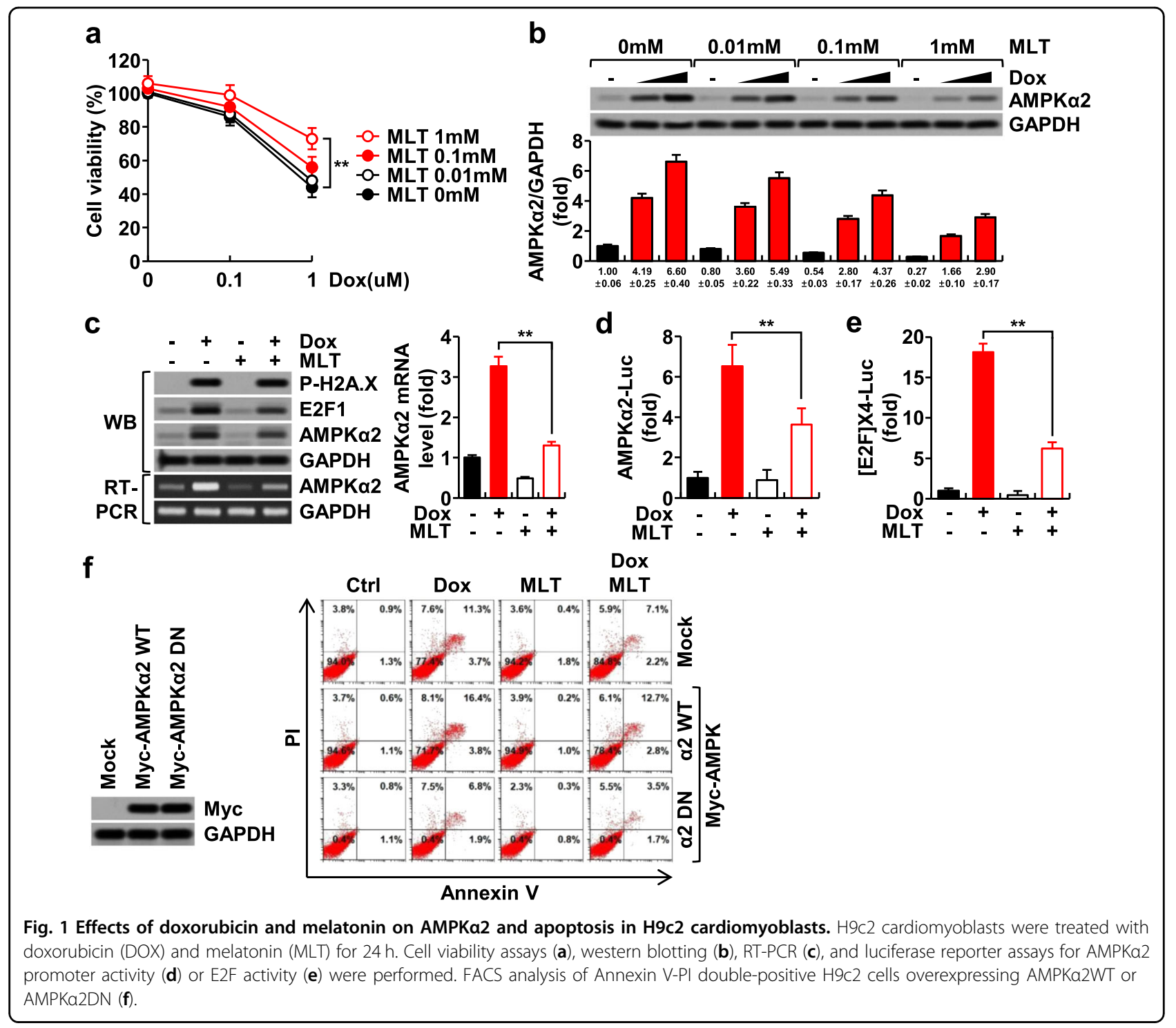

cellular levels of AMPK $\alpha 2$, as well as the levels of AMPK $\alpha 2$ in the mitochondrial fraction, were increased by doxorubicin in the WT and Ampk $\alpha 1^{-1-}$ MEFs, and melatonin significantly reduced the cellular and mitochondrial levels of AMPK $\alpha 2$ (Fig. 3a). Therefore, comparisons of AMPK $\alpha 2-$ positive MEFs (WT and $A m p k \alpha 1^{-/-}$) and AMPK 2 2-negative MEFs (Ampk $\alpha 2^{-/-}$and $\left.A m p k \alpha 1^{-/-} \alpha 2^{-/-}\right)$provide an excellent approach for investigating the specific role of AMPK $\alpha 2$ in the context of doxorubicin treatment. In accordance with a previous result (Fig. 1f), the doxorubicininduced apoptosis and mitochondrial damage were more severe in the AMPK $\alpha 2$-positive MEFs than in the AMPK 2 2-negative MEFs, as demonstrated by annexin V/PI double staining (Fig. 3b), mitochondrial membrane potential (Fig. 3c), cellular and mitochondrial ROS levels (Fig. 3d), and cellular ATP levels (Fig. 3e). Mitochondria are highly dynamic organelles that maintain their structure and function by undergoing fission and fusion with each other. However, during apoptosis, mitochondria often fragment into smaller units, and the imbalance between fusion and fission is closely related to the degree of mitochondrial dysfunction ${ }^{29,30}$. Treatment of AMPK $\alpha 2-$ positive MEFs with doxorubicin caused fragmentation of mitochondrial structures, a phenomenon that was significantly reduced in AMPK 2 -negative MEFs (Fig. 3f). Collectively, these results suggest that, in the context of doxorubicin treatment, a significant portion of AMPK $\alpha 2$ is translocated to mitochondria, disrupting mitochondrial function and morphology and ultimately contributing to apoptosis and that melatonin preserves mitochondrial function and integrity by suppressing AMPK $\alpha 2$ expression (Figs. 2 and 3). 


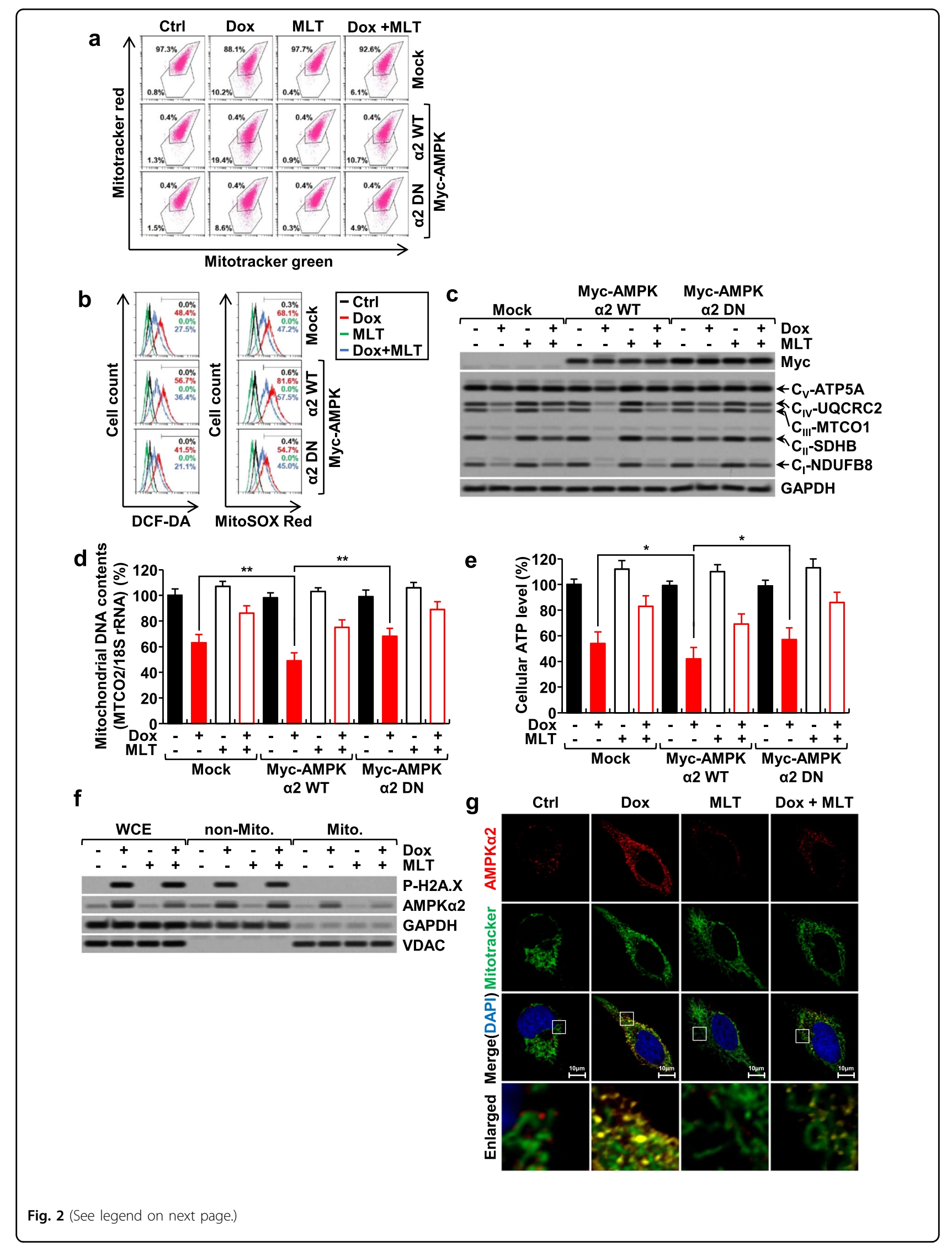


(see figure on previous page)

Fig. 2 Effects of doxorubicin and melatonin on AMPKa2 and mitochondrial function in H9c2 cardiomyoblasts. H9c2 cells overexpressing AMPKa2WT or AMPKa2DN were treated with doxorubicin $(1 \mu \mathrm{M})$ and melatonin $(1 \mathrm{mM})$ for $24 \mathrm{~h}$. Under these conditions, mitochondrial membrane potential (a), cellular and mitochondrial ROS levels (b), mitochondrial OXPHOS complex protein levels (c), mitochondrial DNA contents (d), and cellular ATP levels (e) were measured. The level of AMPKa2 in whole-cell extracts (WCE), nonmitochondrial (non-Mito), and mitochondrial fractions (Mito) (f). Fluorescence images of AMPKa2, mitochondria, and nuclei, which were labeled with Texas Red dye, MitoTracker green, and DAPI, respectively $(\mathbf{g})$.

\section{Melatonin prevents doxorubicin-induced mitochondrial fragmentation by suppressing the activity of mitochondrial AMPKa2}

Despite the findings presented above, it remains unclear whether the enzymatic activity of AMPK $\alpha 2$ is actually critical for doxorubicin-induced mitochondrial damage. Analysis of immunoprecipitated AMPK $\alpha 2$ revealed that treatment with doxorubicin significantly increased its activity, and this phenomenon was effectively blocked by melatonin, as determined by the ratio of phosphorylated (active) AMPK $\alpha 2$ to total AMPK $\alpha 2$ (Fig. 4a). We further measured the mitochondria-specific AMPK $\alpha 2$ activity by transfecting the mito-ABKAR plasmid, which is a mitochondria-specific AMPK activity probe. This construct, which is composed of a mitochondria-targeting sequence, the CFP variant Cerulean, the FHA1 phosphoamino acid-binding domain, an AMPK substrate motif, and the YFP variant cPVE172, is a Förster resonance energy transfer (FRET)-based reporter designed to measure the mitochondria-specific activity of $\mathrm{AMPK}^{31}$ (Fig. 4b). Doxorubicin treatment induced a strong FRET signal, and this effect was significantly attenuated by melatonin (Fig. 4c). We further observed a strong negative correlation between mito-ABKAR FRET signals and mitochondrial length following treatment with doxorubicin and/or melatonin (Fig. 4d). Relevant to our current study, a recent study demonstrated that AMPK phosphorylated mitochondrial fission factor (MFF), a mitochondrial outer-membrane protein that is required for mitochondrial fission, at $\operatorname{Ser}^{14632,33}$. We observed that overexpression of AMPK $\alpha 2 \mathrm{WT}$, but not AMPK $\alpha 2 \mathrm{DN}$, strongly induced the phosphorylation of MFF at Ser ${ }^{146}$ (Fig. 4e). Doxorubicin treatment also strongly induced the phosphorylation of MFF at $\operatorname{Ser}^{146}$, whereas melatonin blocked this phosphorylation (Fig. 4f). To further demonstrate the mitochondria-specific role of AMPK $\alpha 2$, we next used the recently developed construct $\mathrm{mChF}$ Tom20-AIP. This construct was designed to express AMPK inhibitor peptide, termed AIP, which allows for the inhibition of AMPK activity in the mitochondria in a substrate-competitive manner ${ }^{31}$. The transfection of mChF-Tom20-AIP into $\mathrm{H} 9 \mathrm{c} 2$ cells resulted in a significant reduction in the doxorubicin-induced phosphorylation of MFF at Ser ${ }^{146}$, indicating the specific inhibition of $\mathrm{AMPK} \alpha 2$ in the mitochondria (Fig. 4f). Moreover,
mChF-Tom20-AIP significantly blocked doxorubicininduced ROS generation (Fig. 4g), mitochondrial fragmentation (Fig. 4h), and cytotoxicity (Fig. 4i). These results suggest that mitochondria-specific AMPK $\alpha 2$ induces mitochondrial fragmentation in response to doxorubicin and that melatonin attenuates doxorubicininduced cytotoxicity by suppressing mitochondrialspecific AMPK $\alpha 2$ activity and expression.

\section{ROS play a key role in AMPKa2 regulation in the context of doxorubicin and melatonin treatment}

The mechanisms by which doxorubicin modulates AMPK $\alpha 2$ activity or expression are currently unknown. Since doxorubicin induces ROS generation ${ }^{3}$ and AMPK is highly sensitive to intracellular $\operatorname{ROS}^{17}$, we examined whether ROS are involved in the regulation of AMPK $\alpha 2$ in the context of doxorubicin and/or melatonin treatment. Pretreatment with an antioxidant, $N$-acetyl cysteine (NAC), significantly blocked cellular and mitochondrial ROS generation (Fig. 5a), AMPK $\alpha 2$ activation (Fig. 5b), AMPK $\alpha 2$ mRNA transcription (Fig. 5c), and apoptosis (Fig. 5d) in the context of doxorubicin treatment. The effects of melatonin on doxorubicin-induced AMPK $\alpha 2$ activity were almost completely diminished in the presence of NAC (Fig. 5b), suggesting that the effects of melatonin, which attenuates doxorubicin-induced AMPK $\alpha 2$ activation, are largely associated with its strong antioxidant properties. NAC treatment decreased doxorubicin-induced E2F1, AMPK $\alpha 2$, and apoptosis as effectively as melatonin, but a combination treatment of NAC and melatonin showed synergistic suppression of these parameters (Fig. 5c, d). Therefore, in addition to its antioxidant properties, melatonin may affect the signaling pathways upstream of AMPK $\alpha 2$ transcription and apoptosis. To test whether melatonin affects these pathways via its receptor, we examined the effects of the highly selective antagonist 4-P-PDOT on $\mathrm{MT}_{2}$ (melatonin membrane receptor 2) because $\mathrm{H} 9 \mathrm{c} 2$ cells express high levels of $\mathrm{MT}_{2}{ }^{34}$. In the presence of 4-P-PDOT, the effects of melatonin on the doxorubicin-induced levels of AMPK $\alpha 2$, E2F1, and apoptosis markers were significantly reduced (Fig. 5e). Collectively, our data suggest that melatonin inhibits AMPK $\alpha 2$ enzyme activity and expression due to its ROS-scavenging properties while simultaneously suppressing the expression of AMPK $\alpha 2$ and E2F1 via the MT2mediated pathway in the context of doxorubicin treatment. 

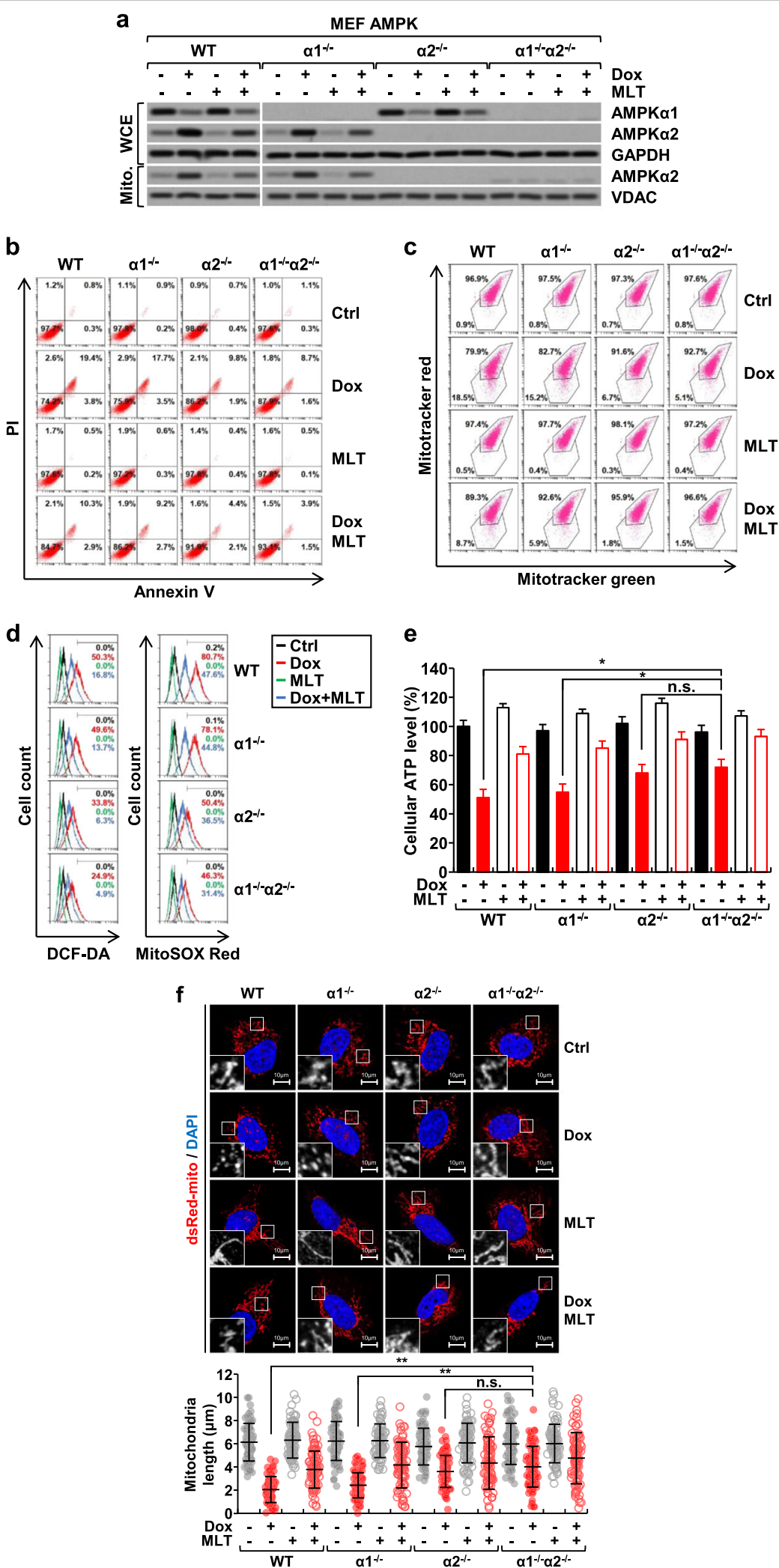

Fig. 3 (See legend on next page.) 
(see figure on previous page)

Fig. 3 Effects of doxorubicin and melatonin on AMPKa2 and mitochondrial function in AMPK knockout MEFs. WT, $\mathrm{Ampka1}^{-1-}, \mathrm{Ampka2}^{-/-}$, and $A m p k a 1^{-1-} a 2^{-1-}$ double-knockout mouse embryonic fibroblasts (MEFs) were treated with doxorubicin (1 $\left.\mu \mathrm{M}\right)$ and melatonin (1 mM) for $24 \mathrm{~h}$. The protein levels of AMPKa1 and AMPKa2 in whole-cell extracts (WCE) and mitochondrial fractions (Mito) (a), apoptosis rate (b), mitochondrial membrane potential (c), cellular and mitochondrial ROS levels (d), cellular ATP levels (e), and mitochondrial length (f) were measured. The pDsRedMito vector was used to label mitochondria (f). n.s., not significant.

The expression level and mitochondria-specific activity of AMPKa2 are tightly associated with cardiotoxicity in mice treated with doxorubicin and melatonin

Hematoxylin and eosin (H\&E) and Masson trichrome staining of cardiac tissues from doxorubicin-treated mice revealed disorganization of muscle fibers, thinner cardiomyocytes, and increased fibrotic areas, including perivascular areas (Fig. 6a). In the doxorubicin-treated group, the expression level of AMPK $\alpha 2$ and rate of TUNEL positivity in cardiac tissues were significantly higher than those in the control group (Fig. 6b). These changes were effectively blocked by melatonin treatment. Western blot analysis of the proteins in cardiac tissue showed that doxorubicin induced the levels of AMPK $\alpha 2$ and P-MFF but reduced the levels of mitochondrial OXPHOS proteins, and these changes were significantly diminished by melatonin treatment (Fig. 6c). Collectively, the in vivo data support the proposed role of AMPK $\alpha 2$ demonstrated in $\mathrm{H} 9 \mathrm{c} 2$ cells in the context of doxorubicin and melatonin treatment.

\section{Discussion}

Doxorubicin effectively kills proliferating cancer cells by intercalating into their DNA and forming covalent adducts. Doxorubicin also accumulates at high concentrations in mitochondria owing to its high affinity for cardiolipin, which is a major phospholipid in the inner membrane of mitochondria; the formation of irreversible interactions between doxorubicin and cardiolipin is known to be an initial cause of mitochondrial dysfunction $^{35}$. This phenomenon is particularly pronounced in cells such as cardiomyocytes, which contain abundant mitochondria. Thus, mitochondrial dysfunction has become a hallmark of doxorubicin-induced cardiotoxicity $^{3,4}$. In addition, doxorubicin activates multiple intracellular signaling pathways that lead to mitochondrial dysfunction and apoptosis ${ }^{35}$. Mitochondria are also known to be a major target of melatonin, which readily crosses all biological membranes because of its amphiphilic nature and thus becomes highly concentrated in mitochondria and nuclei ${ }^{11}$. In addition, melatonin activates multiple signaling pathways that lead to the preservation of mitochondria. It has been reported that melatonin increases the activity of mitochondrial respiratory complexes I and IV, thereby increasing ATP production $^{36,37}$. Melatonin possesses strong ROS- scavenging activity, but it also induces the expression of various antioxidant genes, including glutathione peroxidase, superoxide dismutase, and catalase, while inhibiting the expression of nitric oxide synthase ${ }^{4}$. These beneficial effects of melatonin are expected to effectively mitigate the cytotoxicity of doxorubicin; indeed, the results of various clinical trials suggest that melatonin significantly reduces doxorubicin-induced cardiotoxicity. Thus, the preservation of mitochondrial integrity is a key focus of efforts to develop therapeutic interventions for doxorubicin-induced cytotoxicity ${ }^{10-12}$.

The results of the present study suggest that AMPK $\alpha 2$ is a novel and common target of doxorubicin and melatonin action. In general, $\mathrm{AMPK} \alpha 2$ exhibits a distinctive tissuespecific expression pattern, with high expression in skeletal and heart muscle; it also regulates critical metabolic processes, including glucose tolerance, insulin sensitivity, and weight gain ${ }^{21-23}$. Notably, we observed that AMPK $\alpha 2$ exhibits proapoptotic properties in doxorubicin-treated H9c2 cells and MEFs (Figs. 1f, 3b, 4i), in accordance with our previous report ${ }^{24}$. The proapoptotic nature of AMPK $\alpha 2$ is likely attributable to its activity in mitochondria. Our results showed that doxorubicin regulated AMPK $\alpha 2$ at least at three levels. First, doxorubicin significantly increased AMPK $\alpha 2$ expression at the transcriptional level via E2F1 (Fig. 1), which is a well-known transcription factor that mediates doxorubicin-induced cytotoxicity by inducing a number of genes involved in the apoptotic pathway. Notably, in this context, AMPK $\alpha 2$ was recently shown to be a novel target of E2F1 ${ }^{24}$. Second, a significant portion of AMPK $\alpha 2$ translocated to mitochondria in response to doxorubicin treatment (Fig. 2f, g). Third, doxorubicin increased the mitochondria-specific enzyme activity of $A M P K \alpha 2$, which contributed to mitochondrial dysfunction (Fig. 4). Although the underlying mechanisms by which doxorubicin causes these changes in AMPK are practically unknown, our data suggest that ROS are a critical factor (Fig. 5). Thus, a vicious cycle between ROS and AMPK $\alpha 2$ is likely to contribute to doxorubicininduced cytotoxicity. Specifically, ROS cause multiple changes in AMPK $\alpha 2$ in doxorubicin-treated cells, and AMPK $\alpha 2$, in turn, interferes with mitochondrial integrity, resulting in the generation of additional ROS. The effects of melatonin, which attenuates doxorubicin-induced cytotoxicity, are likely associated with its strong antioxidant properties that suppress the vicious cycle between 

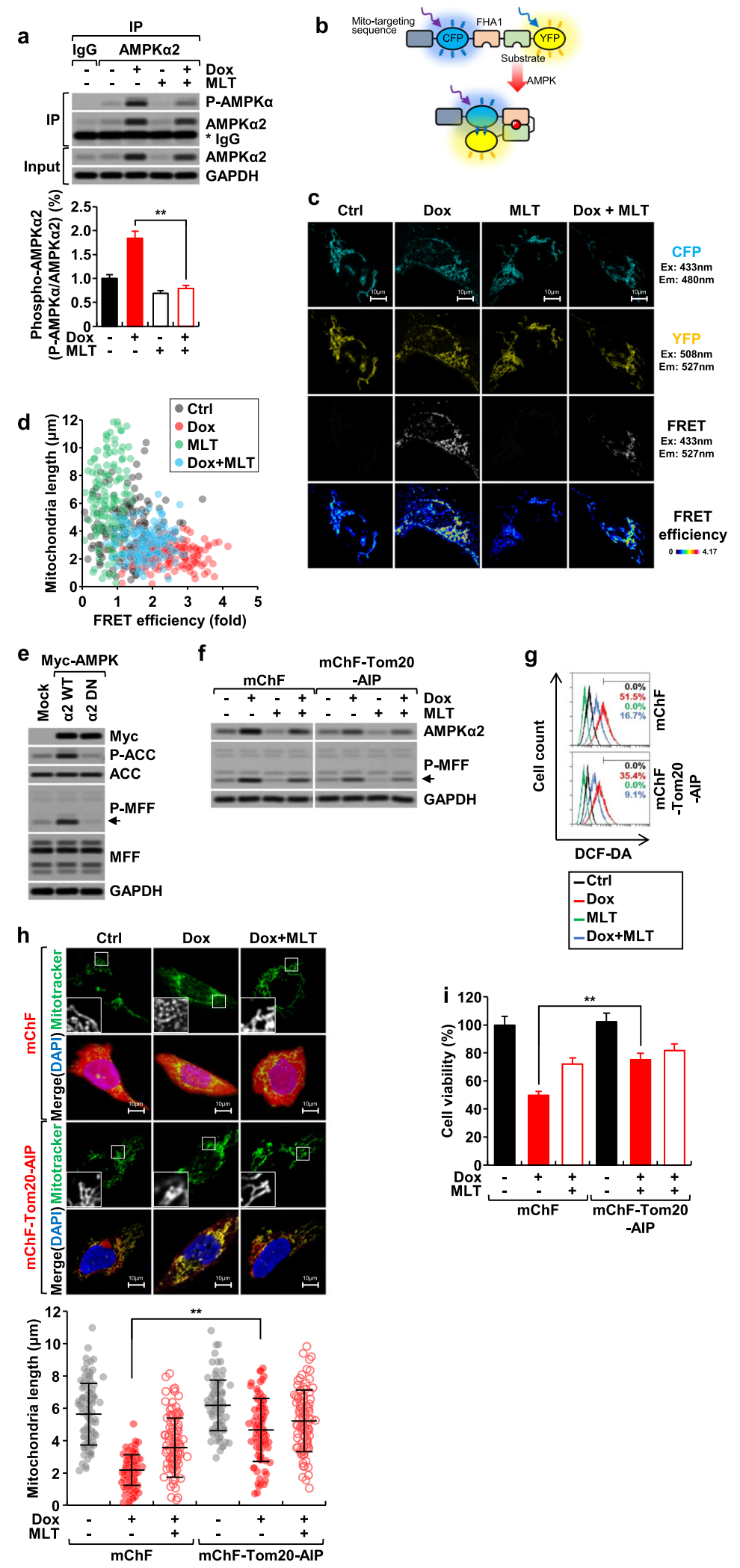

Fig. 4 (See legend on next page.) 
(see figure on previous page)

Fig. 4 Effects of doxorubicin and melatonin on mitochondria-specific AMPKa2 and mitochondrial function in H9c2 cardiomyoblasts. H9c2 cells were treated with melatonin $(1 \mathrm{mM})$ and/or doxorubicin $(1 \mu \mathrm{M})$ for $24 \mathrm{~h}$. a Endogenous AMPKa2 was immunoprecipitated and analyzed by western blotting. $\mathbf{b}$ The structure of mito-ABKAR. c After transfection with the mito-ABKAR plasmid, representative CFP images, YFP images, and FRET images were obtained by confocal microscopy under the indicated conditions. FRET efficiency was represented as pseudocolor images of FRET/CFP. d The correlation between mitochondrial length and FRET signal in the context of melatonin and doxorubicin treatment. e MFF phosphorylated at $\mathrm{Ser}^{146}$ (P-MFF) in H9c2 cells transfected with AMPKa2WT \& DN. P-MFF (f), cellular ROS level (g), mitochondrial length (h), and cell viability (i) were measured in $\mathrm{H} 9 \mathrm{c} 2$ cells transfected with mChF-Tom20-AIP.
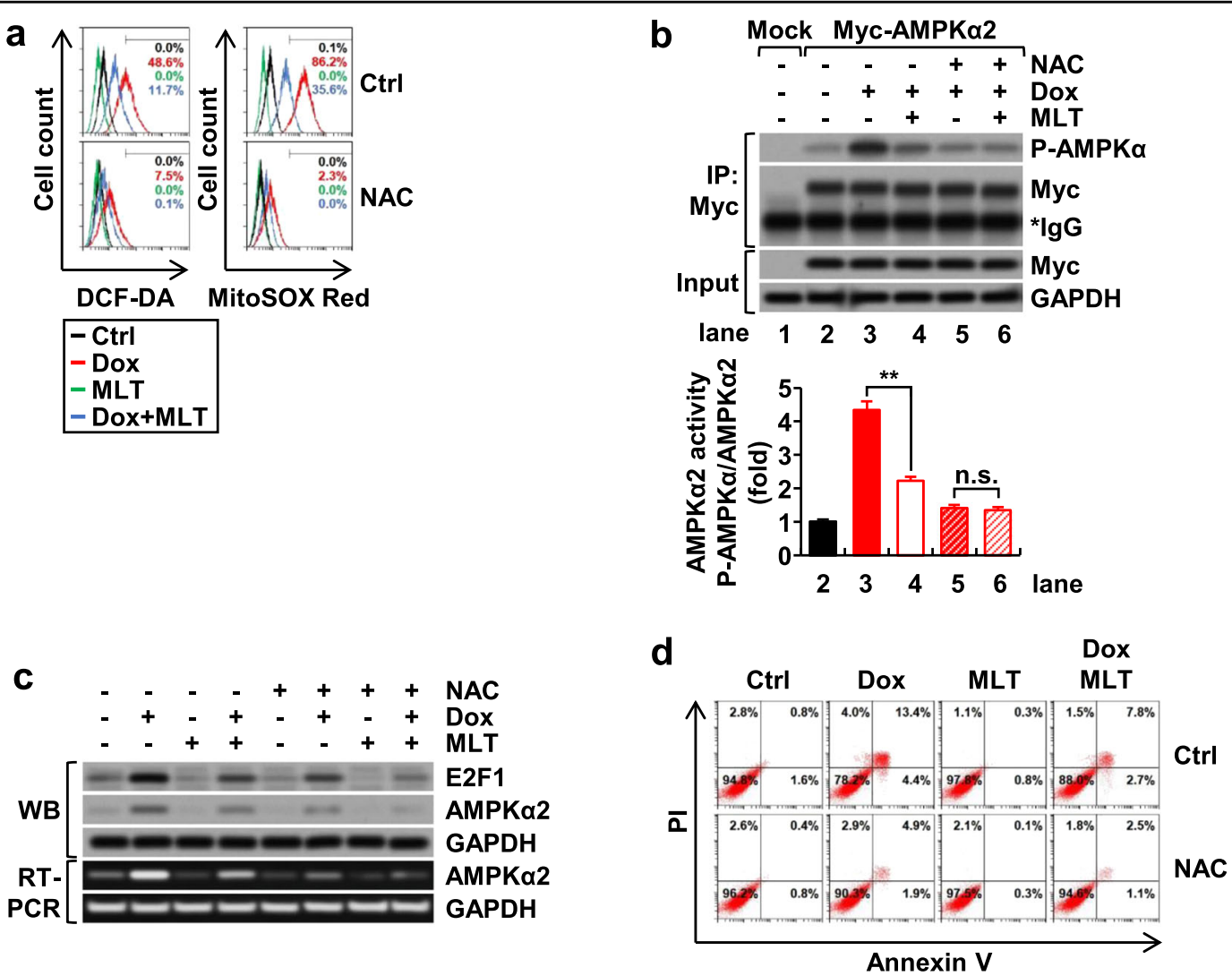

e

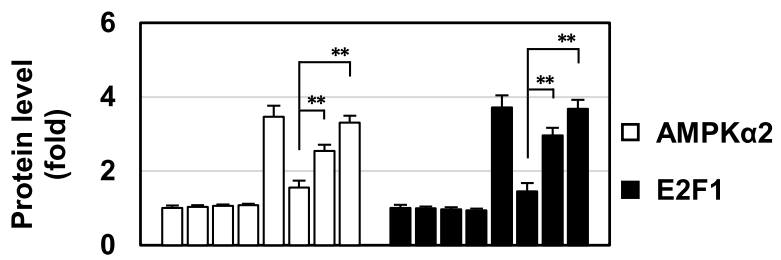

lane 1234567812345678
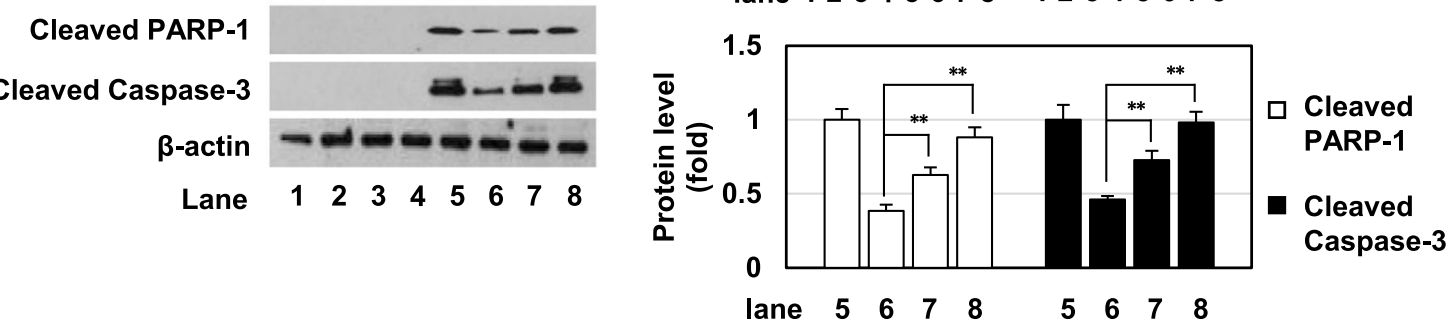

Fig. 5 Role of ROS in the induction of AMPKa2 in the context of doxorubicin and melatonin treatment. H9c2 cells were treated with doxorubicin and melatonin in the presence of NAC $(5 \mathrm{mM})$. Cellular and mitochondrial ROS levels $(\mathbf{a})$, western blot analysis of immunoprecipitated Myc-AMPKa2 after transfection into H9c2 cells (b), western blot and RT-PCR (c), apoptosis rate (d) were measured or performed. e H9c2 cells were treated with doxorubicin and melatonin in the presence of the indicated concentration of 4-P-PDOT, and western blot analyses were performed. 

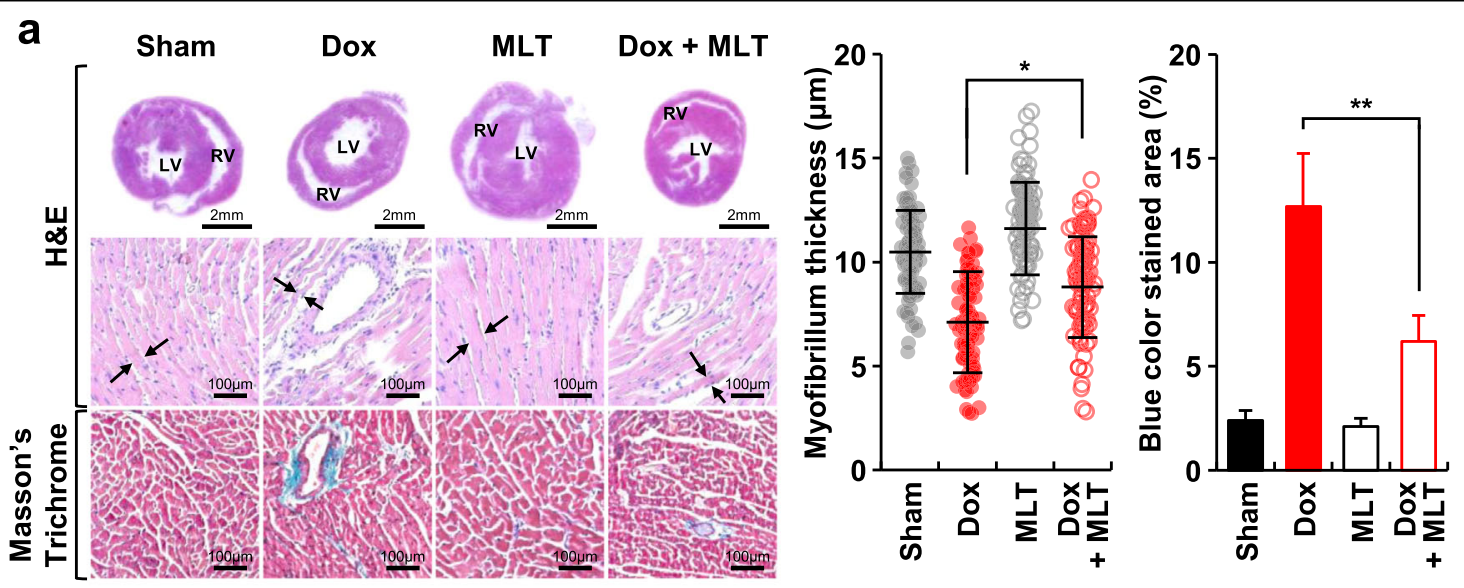

b
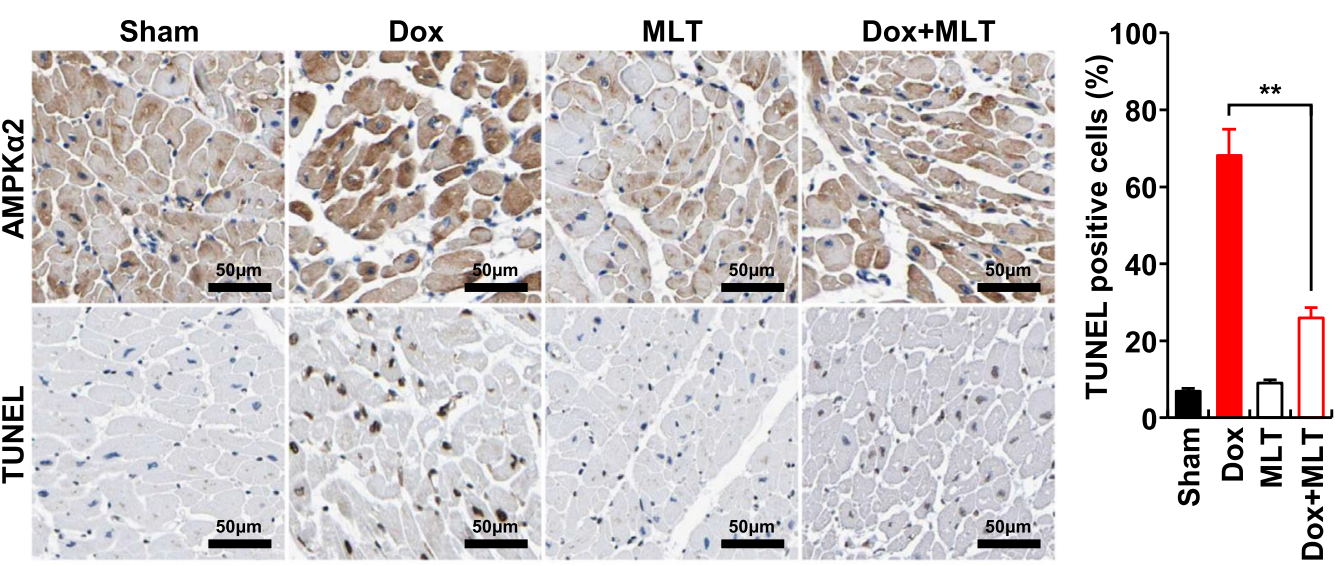

C
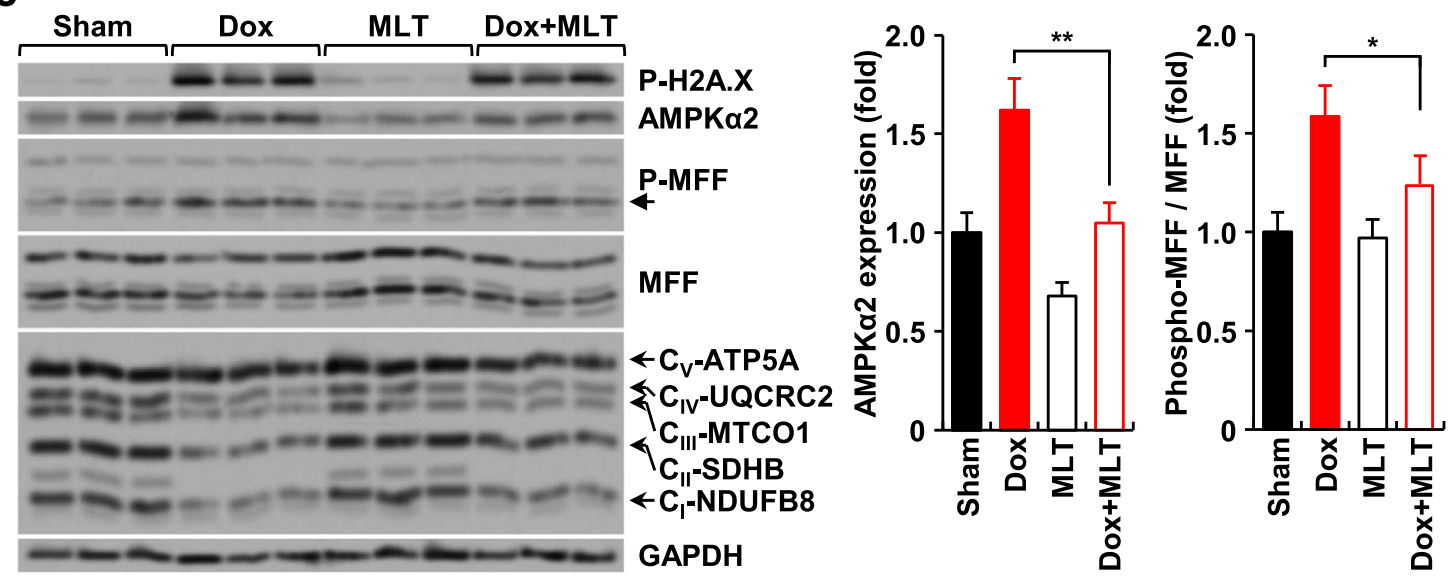

Fig. 6 AMPKa2 in the heart tissue of mice treated with doxorubicin and/or melatonin. Mice were intraperitoneally injected with doxorubicin $(2.5 \mathrm{mg} / \mathrm{kg})$ and/or melatonin $(1 \mathrm{mg} / \mathrm{kg})$ every 2 days for 2 weeks. a After performing the experiments, the heart tissues of the mice were extracted, and H\&E and Masson's trichrome staining were performed. The arrows indicate the thickness of the myofibrillum. $\mathbf{b}$ The expression of AMPKa2 and the number of TUNEL-positive cells in heart tissue were identified by immunohistochemical staining. $\mathbf{c}$ Western blot analysis of the proteins from the heart tissue of each mouse group. Each image and western blot band were quantified using ImageJ software. LV, left ventricle; RV, right ventricle.

ROS and AMPK $\alpha 2$. Additionally, melatonin may regulate the upstream signaling pathways that lead to AMPK $\alpha 2$ transcription via the MT2-mediated signaling pathway.
The relationship between AMPK and mitochondria has been of great interest to researchers in the energy homeostasis field, but most of these previous studies were 
performed without distinguishing among the potentially different roles of the various AMPK isoforms. Therefore, some results appear to be quite contradictory. For example, in contrast to the proapoptotic properties of AMPK $\alpha 2$ described in the current study, a recent publication reported that AMPK $\alpha$, presumably AMPK $\alpha 1$, mediated the protective effects of melatonin against doxorubicin-induced mitochondrial oxidative damage ${ }^{38}$. Another report concluded that AMPK $\alpha 1$ was necessary for the expression of mitochondrial electron transport complex I genes and thereby mitigated metabolic stress and apoptosis in T-ALL cells ${ }^{39}$. More relevant to our current study, mitochondria fission factor (MFF), a mitochondrial outer-membrane protein that is required for mitochondrial fission, was recently identified as a novel substrate for $\mathrm{AMPK}^{32}$. We also observed that doxorubicin and AMPK $\alpha 2$ induced the phosphorylation of MFF at Ser ${ }^{146}$, whereas melatonin blocked this phosphorylation (Fig. 4e, f). Notably, in this context, we observed that the expression level of AMPK $\alpha 2$ increased in response to doxorubicin, whereas that of AMPK $\alpha 1$ decreased, and melatonin treatment resulted in the reversal of each of these expression patterns (Fig. 3a). Therefore, AMPKa isoforms may exert opposing effects by differentially regulating the fate of mitochondria under different conditions. Thus, a clearer understanding of the relationship between AMPK and mitochondria requires the consideration of the isoform-specific roles of AMPK $\alpha$ as well as the mitochondria compartment-specific roles of AMPK. In addition, since the enzyme activity of AMPK $\alpha 2$ can be regulated by doxorubicin and melatonin (Fig. 5b), it would be highly interesting to study how the mechanisms upstream of AMPK, including LKB1 (liver kinase B1) and CaMKK $\beta$ (calcium/calmodulin-dependent protein kinase kinase $\beta$ ), are regulated under these conditions.

Collectively, our data suggest that the preservation of mitochondrial integrity by melatonin is a critical feature of the ability of melatonin to mitigate doxorubicininduced cytotoxicity and that AMPK $\alpha 2$ may serve as a novel target in the design of cytoprotective combination therapies that include doxorubicin.

\footnotetext{
Acknowledgements

This work was supported by grants from the National Research Foundation of Korea (NRF-2018R1D1A1B07049826) and Basic Science Research Program through the National Research Foundation of Korea (NRF) funded by the Ministry of Education (2018R1A6A1A03025124).
}

\section{Conflict of interest}

The authors declare that they have no conflict of interest.

\section{Publisher's note}

Springer Nature remains neutral with regard to jurisdictional claims in published maps and institutional affiliations.
Received: 13 July 2020 Revised: 9 October 2020 Accepted: 28 October 2020.

Published online: 18 December 2020

\section{References}

1. Minotti, G., Menna, P., Salvatorelli, E., Cairo, G. \& Gianni, L. Anthracyclines: molecular advances and pharmacologic developments in antitumor activity and cardiotoxicity. Pharm. Rev. 56, 185-229 (2004).

2. Carvalho, C. et al. Doxorubicin: the good, the bad and the ugly effect. Curr. Med. Chem. 16, 3267-3285 (2009).

3. Tokarska-Schlattner, M., Zaugg, M., Zuppinger, C., Wallimann, T. \& Schlattner, U. New insights into doxorubicin-induced cardiotoxicity: the critical role of cellular energetics. J. Mol. Cell Cardiol. 41, 389-405 (2006).

4. Govender, J., Loos, B., Marais, E. \& Engelbrecht, A. M. Mitochondrial catastrophe during doxorubicin-induced cardiotoxicity: a review of the protective role of melatonin. J. Pineal Res. 57, 367-380 (2014).

5. Minotti, G., Cairo, G. \& Monti, E. Role of iron in anthracycline cardiotoxicity: new tunes for an old song? FASEB J. 13, 199-212 (1999).

6. Singal, P. K., Li, T., Kumar, D., Danelisen, I. \& lliskovic, N. Adriamycin-induced heart failure: mechanism and modulation. Mol. Cell Biochem. 207, 77-86 (2000).

7. Broeyer, F. J. et al. Evaluation of lecithinized human recombinant super oxide dismutase as cardioprotectant in anthracycline-treated breast cancer patients. Br. J. Clin. Pharm. 78, 950-960 (2014).

8. Singh, M. \& Jadhav, H. R. Melatonin: functions and ligands. Drug Disco. Today 19, 1410-1418 (2014).

9. Coto-Montes, A. et al. Role of melatonin in the regulation of autophagy and mitophagy: a review. Mol. Cell Endocrinol. 361, 12-23 (2012).

10. Lissoni, P. et al. Decreased toxicity and increased efficacy of cancer chemotherapy using the pineal hormone melatonin in metastatic solid tumour patients with poor clinical status. Eur. J. Cancer 35, 1688-1692 (1999).

11. Govender, J., Loos, B. \& Engelbrecht, A. M. Melatonin: a protective role against doxorubicin-induced cardiotoxicity. Future Oncol. 11, 2003-2006 (2015).

12. Tengattini, S. et al. Cardiovascular diseases: protective effects of melatonin. J. Pineal Res. 44, 16-25 (2008).

13. Reiter, R. J., Paredes, S. D., Korkmaz, A., Jou, M. J. \& Tan, D. X. Melatonin combats molecular terrorism at the mitochondrial level. Interdiscip. Toxicol. 1, 137-149 (2008).

14. Ramis, M. R., Esteban, S., Miralles, A., Tan, D. X. \& Reiter, R. J. Protective effects of melatonin and mitochondria-targeted antioxidants against oxidative stress: a review. Curr. Med. Chem. 22, 2690-2711 (2015).

15. Yang, Y. et al. A review of melatonin as a suitable antioxidant against myocardial ischemia-reperfusion injury and clinical heart diseases. J. Pineal Res. 57, 357-366 (2014).

16. Hardie, D. G. AMPK: positive and negative regulation, and its role in wholebody energy homeostasis. Curr. Opin. Cell Biol. 33, 1-7 (2015).

17. Hardie, D. G. AMP-activated protein kinase: an energy sensor that regulates all aspects of cell function. Genes Dev. 25, 1895-1908 (2011).

18. Viollet, B. et al. AMPK: Lessons from transgenic and knockout animals. Front Biosci. (Landmark Ed.) 14, 19-44 (2009).

19. Kim, J., Yang, G., Kim, Y., Kim, J. \& Ha, J. AMPK activators: mechanisms of action and physiological activities. Exp. Mol. Med. 48, e224 (2016).

20. Hardie, D. G. AMPK: a target for drugs and natural products with effects on both diabetes and cancer. Diabetes 62, 2164-2172 (2013).

21. Viollet, B. et al. The AMP-activated protein kinase alpha2 catalytic subunit controls whole-body insulin sensitivity. J. Clin. Invest. 111, 91-98 (2003).

22. Villena, J. A. et al. Induced adiposity and adipocyte hypertrophy in mice lacking the AMP-activated protein kinase-alpha2 subunit. Diabetes 53 , 2242-2249 (2004).

23. Jorgensen, S. B. et al. Knockout of the alpha2 but not alpha1 5'-AMP-activated protein kinase isoform abolishes 5-aminoimidazole-4-carboxamide-1-beta-4ribofuranosidebut not contraction-induced glucose uptake in skeletal muscle. J. Biol. Chem. 279, 1070-1079 (2004).

24. Yang, W. et al. AMP-activated protein kinase alpha2 and E2F1 transcription factor mediate doxorubicin-induced cytotoxicity by forming a positive signal loop in mouse embryonic fibroblasts and non-carcinoma cells. J. Biol. Chem. 289, 4839-4852 (2014).

25. Ha, J., Lee, J. K. Kim, K. S., Witters, L. A. \& Kim, K. H. Cloning of human acetylCoA carboxylase-beta and its unique features. Proc. Natl Acad. Sci. USA 93 11466-11470 (1996) 
26. Laderoute, K. R. et al. 5'-AMP-activated protein kinase (AMPK) is induced by low-oxygen and glucose deprivation conditions found in solid-tumor microenvironments. Mol. Cell Biol. 26, 5336-5347 (2006).

27. Lee, J. et al. Hydrogen-rich medium protects mouse embryonic fibroblasts from oxidative stress by activating LKB1-AMPK-FoxO1 signal pathway. Biochem. Biophys. Res. Commun. 491, 733-739 (2017).

28. Zilinyi, R. et al. The cardioprotective effect of metformin in doxorubicininduced cardiotoxicity: the role of autophagy. Molecules 23, 1184 (2018).

29. Chen, H. \& Chan, D. C. Emerging functions of mammalian mitochondrial fusion and fission. Hum. Mol. Genet. 14 Spec, R283-R289 (2005).

30. Liesa, M., Palacin, M. \& Zorzano, A. Mitochondrial dynamics in mammalian health and disease. Physiol. Rev. 89, 799-845 (2009).

31. Miyamoto, T. et al. Compartmentalized AMPK signaling illuminated by genetically encoded molecular sensors and actuators. Cell Rep. 11, 657-670 (2015).

32. Toyama, E. Q. et al. Metabolism. AMP-activated protein kinase mediates mitochondrial fission response energy stress. Science 351, 275-281 (2016).

33. Gandre-Babbe, S. \& van der Bliek, A. M. The novel tail-anchored membrane protein Mff controls mitochondrial and peroxisomal fission in mammalian cells. Mol. Biol. Cell 19, 2402-2412 (2008).
34. Wu, J., Yang, Y., Gao, Y., Wang, Z. \& Ma, J. Melatonin attenuates anoxia/ reoxygenation injury by inhibiting excessive mitophagy through the MT2/ SIRT3/FoxO3a signaling pathway in $\mathrm{H} 9 \mathrm{c} 2$ cells. Drug Des. Devel Ther. 14 2047-2060 (2020)

35. Koleini, N. \& Kardami, E. Autophagy and mitophagy in the context of doxorubicin-induced cardiotoxicity. Oncotarget 8, 46663-46680 (2017).

36. Martin, M. et al. Melatonin-induced increased activity of the respiratory chain complexes I and IV can prevent mitochondrial damage induced by ruthenium red in vivo. J. Pineal Res. 28, 242-248 (2000)

37. Martin, M. et al. Melatonin increases the activity of the oxidative phosphorylation enzymes and the production of ATP in rat brain and liver mitochondria. Int. J. Biochem. Cell Biol. 34, 348-357 (2002).

38. Liu, D. et al. AMPK/PGC1alpha activation by melatonin attenuates acute doxorubicin cardiotoxicity via alleviating mitochondrial oxidative damage and apoptosis. Free Radic. Biol. Med. 129, 59-72 (2018).

39. Kishton, R. J. et al. AMPK Is Essential to Balance Glycolysis and Mitochondrial Metabolism to Control T-ALL Cell Stress and Survival. Cell Metab. 23, 649-662 (2016). 